\title{
Smart Delivery of Multimedia Content for Wireless Applications
}

\author{
Theo Kanter ${ }^{1}$, Per Lindtorp ${ }^{2}$, Christian Olrog $^{3}$, and Gerald Q. Maguire Jr. ${ }^{4}$ \\ $\left\{{ }^{1}\right.$ Theo.Kanter $^{2}$ Per.Lindtorp ${ }^{3}$ Christian.Olrog $\} @$ era.ericsson.se, \\ Ericsson Radio Systems AB, SE-164 80, Stockholm, Sweden. \\ ${ }^{4}$ maguire@ it.kth.se, \\ TeleInformatics, KTH, SE-164 40, Stockholm, Sweden.
}

\begin{abstract}
Packet-oriented access to cellular networks enables us to deliver multimedia content to mobile users. As cellular networks will continue to deliver circuit switched voice for some time to come, care must be taken to avoid interference between these delivery mechanisms, while maximizing the range of services and the number of users. Smart delivery of multimedia content involving agents running in the mobile, the base station and the content provider allows us to dynamically adapt the application and network behavior to each other in order to meet the criteria for specific applications. In particular, this paper examines the delivery of streaming media and interactive voice as Voice over IP (VoIP) to mobile users. Our conclusion is that this, in combination with the dynamic adaptive properties as introduced by the agents, enables us to transfer voice entirely IP over wireless links, thereby freeing further resources for the new applications that we refer to in this paper.
\end{abstract}

\section{Introduction}

Presently, the telecom and datacom industries are converging in different ways. With respect to mobile telephony with GSM, new devices are appearing on the market that integrate data with the telephony voice service in new ways. So-called Smart Phones either include the functionality of an organizer, or can connect wirelessly to an external Personal Data Assistant (PDA) and integrate the functionality of the organizer for smart dialing and messaging with the application that is running in the handset. The Wireless Application Protocol (WAP) is intended to move the point of integration of these services into the cellular access network. WAP-gateways can be used to adapt and convert Internet information, so that the mobile terminal can be used for interacting with a wider range of network-centric services (e.g. electronic payment, subscription to information services, unified messaging, etc.). However, WAP is neither intended nor well suited to transport multimedia content, but rather was targeted at simply extending GSM networks with data services. Only through WAP-gateways can these services be connected to services on the Internet. Therefore, WAP excludes mobile users from directly interacting with Internet content. On the other hand, simple low-bandwidth GPRS (General Packet Radio Service) is introduced in GSM-networks, which will provide direct Internet access to mobile users. GPRS enables the development of multimedia applications for the mobile 
device. These applications can integrate content that resides on the Internet. EDGE (Enhanced Data-rate for GSM Evolution), the successor of GPRS, increases the bitrate and thereby further relaxes the requirements on the mobile applications and Internet content, thus bring even more new multimedia applications to mobile devices. Mobile devices are now able to perform significant computations based on events from various input devices and/or information sources. These events provide information about the user's context and the conditions, which the link is facing. Therefore, applications in mobile devices and nodes in the network that co-operate to deliver multimedia services to users can adapt their mode of communication dynamically based on such events. In such cases, the requirements for the delivery of multimedia IP-content over a link using a wireless access networks are even further relaxed.

\section{Problem Statement}

The important question is therefore: how cleverly can we dynamically shape the applications and Internet content, in order to maximize the delivery of multimedia content to mobile users? Multimedia applications put wide-ranging requirements on links. However, the quality of service (QoS) requirements involved can be categorized by several parameters, which differ in importance for the different type of services (and applications). We examine these parameters below.

\subsection{Latency}

Latency is important for isochronous services, such as voice (e.g., interactive speech) where delays up to 250 milliseconds are perceived as acceptable. Beyond 500 milliseconds the behavior of users gradually adapts itself to the increase in delay and the receiving party usually waits for a ready signal before starting to send. Latency may be due to network-related delays, and buffering in either the application or the device. Latency is not critical in streaming applications, such as Internet radio and other applications that playback multimedia content (e.g. MP3-files) as long as the user is assured that the content is going to be received within a bounded maximum delay.

\subsection{Robustness}

Latency is also important for interactive network games, such as Quake and Unreal, but in this case delay is not correlated to congestion problems. On the other hand, it is important that the packets arrive, otherwise synchronization problems will occur (these are not critical as continuous updates of players' locations are sent). This is normally handled by TCP or by UDP and an application-specific handshake protocol. However, the link level may also provide this service. The interleaving that GPRS does increases the probability that a packet is not lost, but on the other hand the user pays a penalty in increased latency, even if the packet is not lost. 
Ericsson's GPRS Application Alliance has tested the currently most popular network game, Unreal Tournament, with a GPRS simulator with good results [16. Using only one timeslot, the latency (about $300-800 \mathrm{msec}$ ) at $26 \mathrm{kbps}$ incurred by GPRS interleaving does somewhat adversely affect players' performance and appreciation of this application, but not in a critical way.

\subsection{Speech Quality}

The coding/encoding algorithms (codecs) dictate the upper bound of the perceived QoS of multimedia streams (such as speech, video or audio). The lower bound is dictated by the percentage of link packet-loss that the codec is able to tolerate before its performance suffers severely. Modern codecs (e.g., Voxware RT-24) can tolerate up to $30-40 \%$ packet loss, with no additional latency.

\subsection{Requirements}

In order to assure that a certain application is feasible we must show that we can meet the requirements in a satisfactory manner at all times and in a scalable way. Two services of particular interest that will be studied further in this paper are:

1. delivery of streamed audio (e.g., MP3-files, Internet radio stations) and

2. interactive voice - e.g. Internet Telephony

The question is how we should use the functionality in: the mobile device, the wireless link, and the network, in order to provide scalable services and applications, such that the number of users is maximized. When designing our applications, we can use the knowledge that applications have of specific end-user requirements to our advantage, specifically by using strategies that dynamically shape the applications and their use of Internet content. This can be done by carefully dynamically adapting the mobile device, the wireless link, and the network, for each application - such that we can assure that these dynamic adaptations will work in an optimal way. This is not to say that the network access needs to be application dependent. We assume that access to the network is based on IP over the wireless link. Using this IP-access, the application is able to negotiate for its resources. The adaptation software running in the mobile device utilizes local APIs.

A recent paper $|1|$ shows that we can deliver some multimedia content as background IP-traffic over GPRS. In this scheme, special care must be taken to avoid interference with switched voice services - this results in a roughly $30 \%$ underutilization, so as not to hurt the channel planning of the network operators. A successful strategy, which dynamically shapes the application's demands for Internet content, must address a number of issues:

1. We should avoid situations where we would require unnecessary over-provisioning of bandwidth or other network resources in order for the applications to work.

2. We should maximize the number of users that will be able to use the services in a mobile environment.

3. This will make the applications feasible at an earlier point in time, i.e. before network resources are further developed. 
4. Such a strategy will save the network provider cost by avoiding investments in infrastructure that might mean unnecessary over-provisioning, while providing revenue from these new applications and services.

These issues are particularly important regarding IP-access to wireless networks, where a common assumption has been that we must wait until EDGE or W-CDMA is fully deployed before we can start using these new applications.

\section{Proposal}

We propose to use agents to represent the different entities in the mobile device, wireless link, and network. The advantage of such an approach is that the agent can behave intelligently on a local level. For instance, the agent can transform image content from color to black and white in order to reduce the data that needs to be transmitted, based on the device characteristics or the available bandwidth at a given price. In addition, the agents can act intelligently in concert, when a certain application demands resources, they can adjust their behavior depending on a nonlocal context (e.g., routing content to (storage) locations, or to where there are currently wireless connections with high-bit rates available). Agents may even incorporate machine learning mechanisms to improve their performance with respect to QoS over time.

A common objection to the usage of agents is that solutions involving agents require the global adoption of an agent-specific discovery and negotiation schema to make it work successfully. However, [5,6 show how we can avoid this pitfall by relying on a general signaling protocol (e.g., the Session Initiation Protocol - SIP [10|) for the location of resources and use it to set up a session for the entities to do agent negotiations when applicable.

In the following section, we will show how agents can be applied to the mobile device, wireless link, and network, to optimize the QoS parameters (that were mentioned in section 0) for the application.

\subsection{Mobile Device}

The application that runs on the mobile device is modeled as an agent. This software is able to use the local APIs to control the speech codecs, length of the sound buffers, choose between available service classes over the wireless link, and even choose between different wireless links.

Furthermore, in certain situations the application may benefit from roaming to another network node. Since the sending party expects a dialog with the receiving party to continue, even if the receiving party goes off-line, the delivery of content is delayed until the receiving party goes on-line again. Utilizing such knowledge about the purpose of the communication can be important in creating intelligent pushservices.

In addition, an interesting side point is that advance knowledge of transmissions allows the mobile device to go off-line and hence on standby for longer periods of time, without harming the application, but with dramatic improvement in battery life. 


\subsection{Wireless Link}

Adaptation of header compression profiles [11] and lower-level behavior in the wireless link (e.g., coding, etc.) should not be directly visible to the application. On the other hand, we could introduce an entity in the access network, modeled as an agent, which acting as a proxy will select a suitable adaptation on behalf of the application.

\subsection{Network}

In a combined GSM and GPRS base station we may include a content-management agent to monitor unused link frames and keep track of which channels are used for switched voice and which channels are used for packet data. This information can be used to increase the utilization of the available bandwidth for IP-connectivity as compared to the relatively static division planned for such base stations.

Furthermore, we can co-locate a SIP-server with the base station. The role of the SIP-server is to locate the user and certain application resources that (as proposed above) should be modeled as agents. Negotiations of sessions are done using SIP, directly between these entities, without involving a SIP-server.

This way, the content-management agent not only helps the base station to increase its throughput of packet data but can also negotiate with the receiver and sender concerning their needs versus available capacity. This provides a basis for developing strategies to adapt the communication in such a way that it fits the end-user's requirements in an optimal way.

The following sections describe two key multimedia applications and illustrate how the proposed functionality can be used to achieve our goals.

\section{$4 \quad$ Streamed Audio}

Streaming Audio as broadcasted by Internet radio stations, is not sensitive to delays. In fact, seconds or even minutes worth of buffering can be done. The critical part is to gain acceptance from the user for these types of delays. Recently, the downloading and playback of stored music (e.g., MP3) has become very popular. A forthcoming publication [1] shows how stored music can be forwarded to mobile devices as background traffic in GPRS-enhanced GSM-networks. In addition, user audio (e.g., from dictations or classes with hyperlinks to electronic notes) could be uplinked for later playback. With this type of application, it is understood by the end-user that downloading may take considerable time, but this can be acceptable as long as the download time (typically at night or during the workday) does not exceed the period of time between use.

\subsection{Gross vs. Net Content}

An interesting calculation is how many unique bits does a radio station produce per day (eliminating the redundant replays of songs, ads, etc.). Now consider an ensemble 
of radio stations which all play many of the same songs - but have different ads, announcements, and play the songs in a different order — how many unique bits does the ensemble generate? A radio station sending an audio stream at $8 \mathrm{kbps}$ generates roughly $700 \mathrm{Mb}$ during 24 hours. A typical song lasts three minutes. Typically commercials occur four times per hour and last 3 minutes, indicating the station can play 16 songs per hour. This provides room for 384 unique songs but radio station profiling, popularity of certain tunes, and marketing of new music, demand that certain songs appear much more often. Assume, the top ten is played once every two hours, and the next twenty half this rate, and the next ten again at half this rate, hence there will be only 3 random songs per hour. This indicates that the station transmits at best (102 songs * 3 minutes $* 8 \mathrm{kbps}=) 146 \mathrm{Mb}$ of unique bits. As there are very many radio stations playing popular music network providers can save a lot of bandwidth in the backbone by either coordinating transmissions of identical content or pre-caching content in geographically distributed cells (see further section 0) and perhaps only transmit content identifiers.

\subsection{Content Distribution}

The agent in the content-server, the base station and the client can co-operate to adapt the communication in different ways. For instance, the agent in the base station may deduce that several end-users have subscribed to the same content and keep local copies in order to reduce traffic in the backbone. It can also multicast this content when possible. Furthermore, this agent can instruct the base station control software to fill unused frames with data, and also predict unused channels for potentially use of delayed the transmission of content. This requires that client agents running in the mobile should be able to deal with paused or even interrupted transmissions, but these are known and solved issues to many FTP-and other clients today.

Based on communication between the agents in the mobile and in the base station, the agent in the base station can note that some of the content does not have to be sent as all the mobiles in the call which would get it - already have this content in their cache.

In light of this, multicasting "ftp" downloads 17], which allow "data holes", should be investigated. The holes can be filled asynchronous to the main download. This way, a user choosing to download an MP3, which is already being downloaded (say halfway) by another user, can hook in to the existing downstream and fetch the rest separately. The actual delivery may even be delayed intentionally until a given number of subscribers are online, or a timeout is exceeded, introducing a notion of content "launch".

\subsection{Caching}

We may also think in terms of content delivery networks, for situations where radio resources exist, but the GPRS backbone does not have spare bandwidth. This calls for:

1. Web-server replication (geographically separated, co-located with base stations).

2. Reverse (transparent) cached proxying, possibly implementing hierarchical caches. 


\subsection{Buffering}

A calculation of the necessary storage capacity of large buffers in the mobile device shows that you could go for long periods of time without having more than a very low data-rate high-latency background service. For instance $64 \mathrm{MB}$ holds 7 hours of internet radio quality audio [1] - so at the $\sim 32 \mathrm{kbps}$, which is available during the peak of the day (if you could use all of it) - this amount of memory could be filled in 16000 seconds (i.e., roughly 4.4 hours).

The capacity of a macrocell (as configured in 1]) can only supply about 64MB of total transfer during the peak of the day (unless you want to exceed the $2 \%$ call blocking probability). This means you either have to:

1. allocate more capacity to this traffic

2. utilize the unused individual frames within the on-going calls

3. download large portions of the content in hotspots (where you have more available capacity - either because of fewer demands in this cell or because the cell has a higher data rate)

4. download large portions of the content in off peak periods

5. have much larger buffers in the device - so you can pre-load even more content

Dimensioning of buffers is important, it allows us to deploy a Mobile Audio Distribution (MAD) |1 service, where the delay is acceptable as long as you experience continuous audio. It is the user experience or perception, which is important.

Based on the results in section 0 each Internet radio station would send 18.25 $\mathrm{Mb}$ /day, assuming some day-to-day coherence the actual amount is even lower. This means that during the peak of the day has sufficient spare capacity to support more than three such stations even if these stations had to transmit all their content only during the peak voice hours! All this while using 32kbps background capacity.

\subsection{Agents}

Different strategies are possible where companies are able to do directed ads, potentially location or context-aware, paying for the extra cost that this requires in order to have shorter delivery times. Besides adding capacity to either the access network or the device, options 2-3 in section 0 are really the interesting ones where agents are able to help out.

Media files may be streamed from mobile devices to the agent on the access point for subsequent distribution to anyone who desires to listen in. Thus, with GPRS a reporter can collect interviews and broadcast them using at most one channel worth of resources, while simultaneously supporting people listening to MP3-based "stations" on their way home from work. An interesting aspect is when this agent finds superfluous capacity it attempts to pre-order content and trickle it down the wireless link for storage in the mobile.

Furthermore, radio stations often have automated programs, so a network provider could strike a deal with a radio station by buying access to these automatic programming schedules and just multicast the content interspersed with the advertisements to the users at the far end of the network. Exploiting advance knowledge of content programming would save network operators a significant 
investment while offering radio stations and content providers knowledge about what the user likes, thus offering benefits to all parties!

An important goal for research in the field of mobile computing and communication is to build so-called 'unconscious' services: automatic services that do not require user intervention to execute because the components involved are able to make decisions themselves [3.4 5]. An important reason for the use of agents is that they enable us to build such unconscious services. For instance: entering our future homes (where we will have high-bandwidth wireless connectivity between devices) our mobile device will connect to the networked stereo components (where the same information is pre-stored on the server) and hand over the on-going multimedia interaction with the user to it. Before going on a timed standby to save its batteries, the mobile device pre-orders the delivery of a copy of new multimedia content via the server to a content provider, according to the user's current preferences. Agents represent the components mentioned here. These agents only have to demonstrate reasonable local behavior in order to offer a very useful service in concert. The service relieves the user of consciously having to reload the mobile device with fresh content according to the current preferences.

\section{Interactive Voice}

The premise that voice will continue to be the most profitable application, which is upheld by many vendors and operators of cellular networks, is false. Voice can be delivered over IP access 9 10] over GPRS [2] even at bit-rates that will be available in the first generation of GPRS. VoIP over wireless can be delivered at $90 \%$ efficiency (of the used radio spectrum) as compared to switched voice), when smart header compression is applied [11 12]. Previously, we have shown that $1.2 \mathrm{kbps}$ is all that it takes to deliver voice over IP over wireless [2], which is $\sim 10 \%$ of a single GSM voice channel. In further support of the feasibility of VoIP over wireless, DiffServ is a reasonable way of guaranteeing bandwidth for most real-time sensitive applications [1 18] - see further section 0 .

Then, we may ask: how long will the statement remain valid that much of the capacity will be used by switched voice, thus relegating anything else to be delivered in the "spare capacity". Delivering switched voice incurs a high investment and maintenance cost for network operators. For wired access, where there is plenty of bandwidth, the cost imbalance has already overthrown the model of switched voice. The "spare capacity" model will remain valid only until the use of VoIP in cellular networks increases sufficient to trigger a wholesale transition to packet-oriented wireless links. But with the experiment that $1.2 \mathrm{kbps}$ is all that it takes to deliver voice [2], this transition cannot be too long after GPRS is introduced. At this average rate you can support at least 26 simultaneous additional VoIP calls in a macro-cell when during busy hours $\sim 32 \mathrm{kbps}$ of spare capacity is available. Assuming business calling patterns this is sufficient to support a population of hundreds of mobile voice users in the area of the cell. Naturally, there will be a QoS reduction caused by the lower speech quality, but end-users will most likely accept this if it means that voice is delivered essentially free in conjunction with other services. 


\subsection{QoS Considerations}

Statically assigned integrated services should be avoided as much as possible, as it implies switched circuit connections over packet networks, whereas differentiated services are crucial to allow e.g. small VoIP packets to cut through the router's FTP and HTTP queues.

Adaptive "loose" integrated services may be necessary, e.g. monitoring the frequency and recurrence of certain high priority packets, capable of preventing issuance of large size packets which would seriously interfere with the probable next high priority packet.

\subsection{Base Station}

In a recent paper 2] we proposed to use IP directly over the wireless link and thus replace the GSM base station with a router with a radio, thus mobile users will be able to use VoIP for the delivery of interactive voice (see also 78$]$ ). As compared to the scenario in [1 where switched voice is prioritized, this would provide for much more flexible use of the available bandwidth. The following scenario also holds for the combined GSM- and GPRS-base station, but it is clear that the headroom for dynamic intelligent behavior of the access point diminishes as more channels are allocated/dedicated for switched voice traffic.

It is obvious that such a base station with a content-management agent can adapt the transmission of content in a fair profitable way. As above, the access point can be co-located with a SIP-server.

\subsection{Agents}

With respect to VoIP, similar scenarios as mentioned in section 0 apply. However, as VoIP puts other requirements on the mobile device, wireless link, and network, agents can help to further adapt the characteristics of these to the current communication context. For instance, if the available bit rate drops, the agent in the mobile device can renegotiate the codec that is to be used, it may also contact the agent in the base station to temporarily halt the transmission of other content that is not sensitive to delays. In case of packet loss, the agent can suggest a change in header compression profiles [11]. The agent in the mobile device could also negotiate with agents located in other base stations for better conditions and more favorable price per bandwidth and cause the communication to be handed over to one of them. Other scenarios may take in to consideration the context of the user (e.g., when the mobile device contains sensors), affecting the content of the voice content. For instance, the content provider could transmit directional audio for augmented reality applications.

\section{Conclusions}

In telephony applications the load per subscriber during the peak hour corresponds to 3 minutes $/ 60$ minutes $=0.05$ Erlang, where a blocking probability of $1 \%$ can be 
tolerated. Assuming we can transmit 26 simultaneously VoIP sessions (see section 0, means that we can serve $\sim 500$ voice users in a cell just using spare capacity during peak hours. On the other hand we can make reasonable assumptions about user behavior. If users are talking they will be using other services less (e.g., browsing the web or downloading MP3 files). This means a user agent running with the application in the mobile device can mediate between the two applications that were mentioned in this paper. VoIP applications could convey information on speech activity, similar to 'push to talk' by monitoring input to the speech buffers and thereby signal to the multimedia download application to temporarily pause transmissions 14|. This can also be done by statistical prediction and a 'back off' mechanism.

In addition, for streamed audio applications a much higher blocking probability can be tolerated as long as we can show that we have a throughput that ensures that the content is delivered within a bounded maximum period of time (see also section 0 . Above we showed that an agent in the mobile device can mediate on a local level between VoIP and streamed audio download applications. Similarly, the contentmanagement agent can make decisions about the fair distribution of capacity among users of these two applications within the scope of a GPRS/GSM base station through a dialog with the SIP-redirect server. Thereby, the agent has knowledge of ongoing sessions and as mentioned above, can predict traffic and thus assist in planning the transmission of additional Internet content.

The advantage with a agent in the base station is that it can observe the link and utilize the pre-stored bits (MP3, etc.) it has to fill voids in the utilization link - you can have high link utilization but still let voice packets through on time. The same argument regarding monitoring of communication content can be applied to the agent in the mobile device. Speaker dependent speech recognition can be applied using the increased computing capability that is available in mobile devices. A new device first learns "his master's voice". Therefore, voice can not only be surpressed to the point that we send ASCII text "annotated" with voice inflections (consequently causing a further reduction in consumed bandwidth for voice). An agent monitoring content in the mobile device will also be able to alter the mode of communication based upon what is actually being said, which is very useful in applications.

The implications to wireless business models are far-reaching. With GPRS, we have seen that customers get direct access to Internet content. As a consequence, third parties (meaning basically anybody - from private persons, local organizations, to radio- and television broadcasting companies) can provide content to end-users. This means that the network operator has no unique role, unlike the case with WAP gateways. Instead the network operator must seek a new role besides providing competitive price per unit of bandwidth for access to Internet, by offering intelligent support for smart delivery of multimedia content to mobile users. This means providing added value to end-users. At the same time, hosting of Internet content and smart transmission to mobile users creates a business opportunity for network operators to offer these services to content providers. Network operators offering agent hosting can also sell their end-users as a potential audience to those who want to send commercials.

In this paper, we proposed to use agents to represent the different entities in the mobile device, wireless link, and network (that can either behave intelligently on a local level or act intelligently in concert, based on a non-local context). By numerous examples we have demonstrated how this approach can be successfully applied to dynamically shape the applications and Internet content, in order to maximize the 
delivery of multimedia content to mobile users, by taking into account the context of the users and the conditions of the communication. In addition, the transferal of switched voice to VoIP further frees resources for the dynamic shaping of applications and our conclusion is that this transition will take place not too long after GPRS is introduced.

In conclusion, we have demonstrated by our approach of smart delivery of multimedia content in wireless that we can:

1. avoid situations where we would require unnecessary over-provisioning of bandwidth or other network resources in order for the applications to work.

2. maximize the number of users that will be able to use the services in a mobile environment.

3. make the applications feasible at an earlier point in time, i.e. before network resources are further developed, and

4. that such a strategy will save the network provider cost by avoiding investments in infrastructure that might mean unnecessary over-provisioning, while providing revenue from these new applications and services.

\section{Future Work}

Future work will look at the specifics of the interaction between the SIP-server, the content management agent in the base station, the agent located at the content provider, and the agent in the mobile device. Furthermore we will study how the agent in the mobile device can interact with communication resources, in particular those governing VoIP QoS, e.g. DiffServ and mobility. Another issue that needs to be addressed concerns how the content-management agent interacts with the radio resources in the base station.

\section{References}

1 P. Lindtorp, "Utilizing Spare Capacity in Radio Access Networks", Master Thesis, School of Electrical Engineering and Information Technology, Royal Institute of Technology, Sweden, December 1999.

2 T. Kanter, C. Olrog, G. Maguire, "VoIP over Wireless for Mobile Multimedia Applications", Personal Computing and Communication Workshop, November 1999.

3 T. Kanter, C. Frisk, H. Gustafsson - "Context-Aware Personal Communication for Teleliving”, Personal Technologies (vol. 2 issue 4, 1998: p. 255 - 261).

4 T. Kanter and H. Gustafsson, "VoIP in Context-Aware Communication Spaces", Proceedings of International Symposium on Handheld and Ubiquitous Computing (HUC 99), Oct. 1999.

5 T. Kanter and H. Gustafsson, "Active Context Memory for Service Instantiation in Mobile Computing Applications," Proceedings of the Sixth IEEE International Workshop on Mobile Multimedia Communications (MoMuC'99), Nov. 1999, p. 179-183. 
6 T. Kanter, “Adaptive Personal Mobile Communication”, forthcoming Licentiate Thesis, Dept. of Teleinformatics, Royal Institute of Technology, Sweden.

7 C. Olrog - "GSM SoftModem on Linux, a Direct Radio Link Protocol Interface", Master Thesis, Dept. of TeleInformatics, Royal Institute of Technology, Sweden, April 1999.

8 T. Turletti, H. Bentzen and D.L. Tennenhouse, "Towards the Software Realization of a GSM Base Station", IEEE/JSAC, Special Issue on Software Radios, Vol. 17, No. 4, pp. 603-612, April, 1999.

9 ITU-T Recommendation H.323 v2 (1998) Packet Based Multimedia Communication Systems.

10 M. Handley, H. Schulzrinne, E. Schooler, J. Rosenberg - RFC 2543 on SIP: Session Initiation Protocol, IETF/Network Working Group - March 1999.

11 L-E. Jonsson, M. Degermark, H. Hannu, K. Svanbro, "RObust Checksum-based header COmpression" (ROCCO), IETF Network Working Group Draft, September 1999.

12 M. Engan, S. Casner, C. Bormann - RFC 2509 on IP Header Compression over PPP, IETF/Network Working Group, February 1999.

13 J. Mitola III, Cognitive Radio, Licentiate Thesis, Dept. of Teleinformatics, Royal Institute of Technology, Sweden, Sept. 1999.

14 J. Mitola III, "Cognitive Radio for Flexible Mobile Multimedia Communications," Proceedings of the Sixth IEEE International Workshop on Mobile Multimedia Communications (MoMuC'99), Nov. 1999, p. 3-10.

15 V. G. Bose, "The Impact of Software Radio on Wireless Networking," Mobile Computing and Communications Review, Volume 3, No. 1, January 1999.

16 News articles on www.planetunreal.com, "UT Over Wireless" and "UT Over GPRS", 11/19/99.

17 J. Ioannidis, G. Q. Maguire Jr., "The Coherent File Distribution Protocol," RCF 1235, IETF Network Working Group.

18 S. Black, D. Black, M. Carlson, E. Davies, Z. Wang, W. Weiss, “An Architecture for Differentiated Services,” RCF 2475, IETF Network Working Group 